\title{
Construção e Validação de um Instrumento para Avaliar Auto-Eficácia em Situações de Treinamento, Desenvolvimento e Educação de Pessoas
}

\author{
Development and Validation of an Instrument to Assess Self-Efficacy \\ in Personnel Training, Development and Education Situations
}

\author{
Pedro Paulo Murce Meneses* \& Gardênia da Silva Abbad \\ Universidade de Brasília
}

\begin{abstract}
Resumo
Pesquisas desenvolvidas nas últimas décadas indicam a relevância de características individuais, como auto-eficácia, na explicação de efeitos de programas educacionais. Inúmeras medidas específicas de auto-eficácia foram elaboradas e validadas neste ínterim. Entretanto, estas escalas confundem-se com medidas que avaliam os efeitos de ações educacionais sobre o desempenho dos indivíduos. São raros os estudos que desenvolveram escalas gerais de auto-eficácia. O presente estudo relata o desenvolvimento de uma escala de auto-eficácia geral. O instrumento, composto por 15 itens, foi submetido à validação teórica e à validação empírica. Neste último processo, 1845 treinandos participaram da pesquisa, de forma que suas respostas foram submetidas a análises fatoriais exploratórias. Os resultados indicaram a validade, a precisão e a confiabilidade do instrumento de auto-eficácia desenvolvido.

Palavras-chave: Auto-eficácia; Característica da clientela; Variáveis auto-referentes; Avaliação de treinamento.
\end{abstract}

\begin{abstract}
Researches done in the last decades suggest the importance of individual characteristics, such as selfefficacy, to explain the effects of training programs. Several specific measures of self-efficacy were developed and validated. These scales are confounded with impact measures that evaluate post-training individual performance. Studies concerning the development of self-efficacy generic measures are still rare. This study reports the development of a self-efficacy generic scale. The instrument, made up of 15 items, was submitted to a theoretical validation as well as to an empirical validation. In this last validation process, 1845 trainees participated of the research. Their answers were submitted to exploratory factor analysis, and the results indicated the validity, precision, and reliability of the self-efficacy instrument which was developed.

Keywords: Self-efficacy; Trainees' characteristics; Self-referent variables; Training evaluation.
\end{abstract}

Impelidas pela crescente competitividade, resultante, entre outros aspectos, dos recentes avanços nos meios de transporte e de comunicação, as organizações passam a exigir das pessoas maior nível de qualificação e um conhecimento mais global do processo produtivo. Neste contexto, como previsto por Goldstein (1991) há mais de 15 anos, programas de treinamento, desenvolvimento e educação (TD\&E) passaram a representar parcela significativa dos investimentos organizacionais para geração de diferenciais competitivos. Estimativas recentes

${ }^{*}$ Endereço para correspondência: SQN 303, Bloco H, apto. 112 Brasília, DF, Brasil, CEP 70745-080. Tel.: +(61)3327 0330. E-mail: pemeneses@yahoo.com.br Os autores agradecem à Prof. Dr. ${ }^{a}$ Maria Júlia Pantoja pelas importantes contribuições para o desenvolvimento deste artigo, sobretudo no tocante à revisão de literatura ora apresentada. sugerem que os gastos com esses tipos de programas, nos Estados Unidos, alcançam a cifra de 200 bilhões de dólares (Salas \& Cannon-Bowers, 2001). Apesar dos consideráveis investimentos, de acordo com os autores supracitados, apenas uma pequena parcela, aproximadamente $10 \%$, parece contribuir para a melhoria dos desempenhos individuais e organizacionais.

Em busca de respostas para este inexpressivo retorno, pesquisadores e profissionais voltaram-se para o estudo do impacto de uma série de variáveis sobre resultados de programas de TD\&E, principalmente em termos de desempenho individual pós-treinamento. Justamente devido a pesquisas desta natureza é que a área de TD\&E foi elevada a tópico de interesse mundial (Philips \& Philips, 2001) ou mesmo alcançou o status de ciência (Freitas \& Borges-Andrade, 2004). Brevemente, pode-se dizer que essas pesquisas reconheceram, no decorrer das últimas três décadas, a influência do contexto organizacional 
sobre os efeitos dos programas de treinamento (e.g. Abbad, 1999; Britto, 1999; Coelho, 2004; Meneses, 2002; Sallorenzo, 2000; Tamayo, 2002; Tannenbaum \& Yukl, 1992; Zerbini, 2003).

Além de variáveis relacionadas ao contexto organizacional, outra linha de pesquisa em avaliação de TD\&E tem investigado a influência de características individuais - sócio-demográficas, psicossociais, motivacionais e cognitivo-comportamentais - sobre resultados de treinamento. Entre essas características individuais, merece destaque a auto-eficácia, devido a evidências, provenientes de inúmeras pesquisas nacionais e internacionais (e.g. Abbad, Pilati, \& Pantoja, 2003; Salas \& Cannon-Bowers, 2001), de que essa característica constitui importante variável explicativa de resultados individuais de treinamento.

De fato, a análise da literatura sugere que auto-eficácia associa-se pelo menos a um dos três níveis de avaliação de treinamento propostos por Kirkpatrick (1976): reação, aprendizagem ou transferência (e.g. Abbad et al., 2003; Brown \& Latham, 2000; Morin \& Latham, 2000; Warr, Allen, \& Birdi, 1999). Além disso, crenças de autoeficácia parecem atuar como variável moderadora na aplicação de conhecimentos e habilidades adquiridos em programas de treinamento.

No que se refere ao desenvolvimento de medidas, embora alguns autores, como Brockner (1988), distingam auto-eficácia geral e específica, a maioria dos estudos tem focalizado a elaboração e validação de escalas específicas que medem a crença auto-referente sobre o quanto um indivíduo se sente capaz de realizar com sucesso determinadas tarefas, tal como aquelas ensinadas em treinamentos (e.g. Ford, Quiñones, Sego, \& Sorra, 1992; Maurer \& Pierce, 1988; Page \& Hulse-Killacky, 1999; Tannenbaum, Mathieu, Salas, \& Cannon-Bowers, 1991). Ainda são raros na literatura estudos que enfocam o desenvolvimento de medidas de auto-eficácia contextualmente desvinculadas.

Apesar da constatação, escalas desta natureza, por não se aterem a uma situação específica, podem contribuir mais rapidamente para a sistematização de conhecimentos acerca das relações entre cognições e realizações individuais. Desta forma, o presente estudo relata o desenvolvimento e a validação de uma escala de auto-eficácia geral em contexto de treinamento. Anteriormente à descrição deste processo de concepção, entretanto, é apresentada uma breve revisão de literatura sobre o conceito e medidas de auto-eficácia.

\section{Revisão de Literatura}

Auto-eficácia é um conceito derivado da Psicologia Social Cognitiva que vem recebendo, mais recentemente, atenção crescente de teóricos e pesquisadores do campo da Psicologia Organizacional. De acordo com Bandura (1989), refere-se às crenças do indivíduo em suas próprias capacidades para mobilizar motivação, recursos cognitivos e cursos de ação necessários ao sucesso das ações em que se engajam. Supõe-se que, ao se perceberem capazes de obter sucesso em suas atividades, os indivíduos tendem a apresentar melhores níveis de desempenho em relação àqueles que, contrariamente, julgam-se incapazes.

Nessa definição, estão envolvidos três importantes aspectos: (a) auto-eficácia pode compreender o desempenho de uma tarefa específica, visto que informações sobre o indivíduo, as características da tarefa e o contexto de trabalho, por exemplo, podem contribuir para uma avaliação mais precisa do indivíduo acerca de suas próprias capacidades; (b) auto-eficácia é um construto dinâmico, composto por julgamentos que podem mudar ao longo do tempo em função da aquisição sistemática de novas informações e experiências; e (c) as crenças de auto-eficácia refletem um processo individual complexo, que envolvem a criação e o teste de formas alternativas de comportamentos e de estratégias de esforço perseverante.

A partir da definição e da base teórica referente, de base cognitivista, o conceito de auto-eficácia tem sido estudado em diversos contextos, como relatam Azzi e Polydoro (2006). Entre as inúmeras aplicações do construto, destacam-se pesquisas na área de Psicologia, Educação, Saúde e Esporte. Por exemplo, os autores discutem a importância do uso de instrumentos de auto-eficácia a fim de permitir que alunos auto-regulem (e.g. estratégias cognitivas de aprendizagem) seus próprios processos de aprendizagem. Apesar de sua enorme aplicação, o presente estudo foi desenvolvido em situações de treinamento, de forma que a seguir será dada ênfase à aplicação e a importância do conceito em situações desta natureza.

Com base na definição proposta por Bandura (1989) e ainda na análise de artigos teóricos e empíricos que focalizam auto-eficácia e suas relações com resultados de treinamento (e.g. Eden \& Kinnar, 1991; Ford et al., 1992; Gist, 1989; Gist \& Mitchell, 1992; Latham \& Frayne, 1989; Mathieu, Martineau, \& Tannenbaum, 1993; Noe, 1986; Sherer \& Adams, 1983; Tannenbaum et al., 1991), auto-eficácia é definida, no presente estudo, como crenças gerais dos indivíduos acerca de suas capacidades de obter sucesso.

Apesar da aparente precisão da definição adotada neste artigo, o conceito de auto-eficácia frequentemente se sobrepõe, na literatura científica, a outros construtos auto-referentes, essencialmente com autoconceito, autoimagem e auto-estima. Entretanto, vale enfatizar que esses conceitos já foram definidos por outros autores, o que permite estabelecer uma diferenciação entre os mesmos. Autoconceito consiste em um conjunto de atitudes e crenças inter-relacionadas, que um indivíduo tem a respeito de si próprio, decorrentes da maneira como se percebe (Lummertz \& Biaggio, 1986). Auto-imagem, por sua vez, é sinônimo de autoconceito, porém enfatiza o aspecto social. Por outro lado, auto-estima refere-se a 
uma atitude valorativa (Gist \& Mitchell, 1992) com ênfase na satisfação ou insatisfação do indivíduo consigo mesmo. Pode-se afirmar, portanto, que auto-eficácia é um aspecto do autoconceito, diferenciando-se de autoestima, uma vez que envolve basicamente processos de natureza cognitiva e não é inerentemente avaliativo (Bandura, 1989).

Expectativa de resultados, conforme definido na Teoria de Expectância (Vroom, 1995), também é um conceito que tem sido considerado similar ao de auto-eficácia. Segundo Bandura (1989), porém, expectativas de resultados implicam no julgamento do indivíduo acerca das prováveis conseqüências que determinado comportamento irá produzir, enquanto que auto-eficácia consiste na expectativa de um único tipo de conseqüência, a saber: ou sucesso, ou fracasso. Mas é preciso notar que os resultados não ocorrem como eventos desarticulados das ações. A maioria dos resultados decorre de ações empreendidas pelo indivíduo e, conseqüentemente, seus comportamentos determinam em grande parte as experiências de resultados. Similarmente, os tipos de resultados que o indivíduo antecipa dependem fortemente de seus julgamentos acerca de suas próprias capacidades para desempenhar uma determinada tarefa. Apesar de poder acreditar que uma ação particular produzirá determinado resultado, segundo a definição de auto-eficácia utilizada neste estudo, o indivíduo não age com base nessa crença, pois ele questiona se realmente é capaz de executar as atividades necessárias ao seu alcance.

Entre esses questionamentos, a complexidade da tarefa cognitiva pode aumentar a probabilidade de erros de avaliação (Bandura, 1988). Além disso, o tempo entre a avaliação de auto-eficácia e avaliação do critério pode diminuir a predicabilidade da auto-eficácia, conforme demonstrado por Wood e Locke (1987). Outro aspecto que parece contribuir para degradar o relacionamento existente entre auto-eficácia e desempenho é o grau de ambigüidade do feedback. Ainda, quanto menos precisas forem as definições sobre os níveis de desempenho da tarefa, menores as correlações entre o construto e o desempenho observado. Finalmente, quanto menor a experiência do indivíduo na execução da tarefa, tanto menor a precisão da predição do desempenho.

O problema que se apresenta para o pesquisador, portanto, é determinar se as baixas correlações entre autoeficácia e desempenho se devem à complexidade da tarefa, ao método de avaliação (quando e como a autoeficácia foi avaliada) ou à familiaridade do indivíduo com a tarefa em questão. Na prática, tentativas de melhorias do desempenho no trabalho têm focalizado o aprimoramento das tarefas (desenho, do trabalho e condições de trabalho), uso de técnicas motivacionais e de gestão (estabelecimento de objetivos, reforçamento, feedback, etc.) e melhorias nas habilidades por meio de programas de TD\&E. No entanto, cabe destacar o papel moderador que a auto-eficácia pode exercer sobre os efeitos dessas mu- danças no desempenho. De fato, evidências empíricas têm demonstrado que a auto-eficácia influencia o grau de aquisição de habilidades e retenção em situações de aprendizagem (e.g. Gist, 1989). Neste sentido, esforços empreendidos pelas organizações de trabalho para melhoria das habilidades de seus profissionais, por meio de ações de TD\&E, podem ser facilitados pelo entendimento e implementação de estratégias voltadas para o desenvolvimento da auto-eficácia.

Conforme revisão de literatura realizada por Salas e Cannon-Bowers (2001), o conceito de auto-eficácia, largamente estudado na última década, assume grande importância em treinamentos à medida que propicia melhores resultados de aprendizagem e desempenho pós-treinamento. De acordo com revisão realizada por Borges-Andrade (1997), inúmeras pesquisas apontam para auto-eficácia como importante variável explicativa de sucesso dos participantes em treinamentos de diferentes domínios e atividades.

Tannenbaum e Yukl (1992) resumem diversos resultados de pesquisas sobre o conceito em questão. Autoeficácia pode ser considerada um antecedente em potencial de eficácia de treinamentos, pois indivíduos com índices elevados de auto-eficácia tendem a apresentar melhores níveis de desempenhos, ainda em treinamento, do que indivíduos com baixa auto-eficácia (e.g. BouffardBouchard, 1990; Taylor, Locke, Lee, \& Gist, 1984). Indivíduos que deixam treinamentos com a crença de que podem desempenhar com sucesso a tarefa na qual foram treinados, provavelmente resistem mais ao encontrarem obstáculos ao uso das novas habilidades no trabalho (Marx, 1982).

Mathieu et al. (1993) enfatizam o relacionamento significativo de índices de auto-eficácia ao final de treinamentos com medidas de transferência pós-treinamento e desempenho no trabalho. Interessados no desenvolvimento de auto-eficácia durante treinamentos e no relacionamento desse desenvolvimento com múltiplas medidas de resultados de treinamento, os autores obtiveram resultados valiosos. Entre eles, auto-eficácia não estaria relacionada com reações e limitações situacionais ao uso das novas habilidades no trabalho.

Colquit, LePine e Noe (2000) ressaltam que auto-eficácia tem-se mostrado positiva e fortemente relacionada a desempenho no trabalho. Em situações de treinamento, o relacionamento entre auto-eficácia e escolha, esforço e persistência na realização da tarefa torna mais provável o estabelecimento de uma relação positiva entre auto-eficácia e resultados de treinamento. No estudo conduzido pelos autores, auto-eficácia relacionou-se, entre outros, com motivação para aprendizagem $(r=0,42)$, transferência do treinamento $(r=0,47)$, conhecimento declarativo $(r=0,30)$, aquisição de habilidades $(r=0,32)$, desempenho no trabalho $(r=0,22)$ e reações $(r=0,17)$.

Como observado, auto-eficácia assume posição importante na determinação de eficácia de treinamentos. Para 
Cervone e Scott (1995), o detalhamento dos mecanismos pelos quais as percepções de auto-eficácia afetam resultados comportamentais evitaria a presença de modelos desconhecidos de desempenho humano, onde permanecem incógnitos processos que ligam cognições a realizações. Em situações de TD\&E, um dos guias para a escolha do que será avaliado é a escassez de tempo hábil que os participantes dispõem para responder a grandes quantidades de questionários e itens.

Como a maioria dos instrumentos de auto-eficácia apresenta um número elevado de itens e ainda é direcionada para a mensuração de impactos específicos de treinamentos em atitudes dos treinandos, buscou-se desenvolver, neste estudo, um instrumento genérico e de tamanho reduzido. Além de facilitar a condução de pesquisas e de acelerar a sistematização de conhecimento acerca da influência da auto-eficácia na geração de resultados individuais de TD\&E, a proposição de um instrumento que avalie a auto-eficácia como disposição geral do indivíduo pode auxiliar, ainda, na classificação de resultados de treinamentos, de modo que seja possível agrupar eventos instrucionais pela intensidade e abrangência de seus efeitos sobre auto-eficácia. O processo de construção e validação deste instrumento de auto-eficácia geral é relatado em seguida.

\section{Método}

Os itens que compuseram a primeira versão do instrumento foram elaborados a partir da definição de Bandura (1989), segundo o qual auto-eficácia refere-se a crenças sobre as próprias capacidades individuais de obter sucesso na realização de determinada tarefa e envolve, entre outros aspectos, a criação e o teste de formas alternativas de comportamentos e de estratégias de esforço perseverante. Além disso, é importante lembrar que o conceito integra a Teoria de Aprendizagem Social do autor referido, segundo a qual, em linhas gerais, a auto-eficácia se desenvolve a partir de sucessivos processos de interação.

Neste sentido, foram construídos os itens constituintes do instrumento de auto-eficácia geral, relativos: aos autojulgamentos acerca das próprias capacidades/habilidades; à motivação para ação; ao enfrentamento de obstáculos/ persistência; à predisposição ao desafio; à abertura à experiência; à capacidade de recuperação diante de fracassos; à influência do sucesso inicial para o sucesso final de uma atividade; ao histórico de sucessos anteriores; e à necessidade de encorajamento externo/instruções.

Desta forma, o instrumento de auto-eficácia geral, em um primeiro momento, englobou 15 itens que aferiam a percepção geral do participante do treinamento acerca de sua capacidade para realizar determinadas atividades com sucesso. Estes itens estavam associados a uma escala do tipo Likert, de concordância, contendo 5 pontos, em que o valor 1 representava a total discordância do participante e o valor 5, a total concordância. Constituídos os itens, a escala de respostas, as instruções e a for- ma do instrumento, realizou-se uma validação semântica com 15 servidores públicos de três empresas do Distrito Federal (DF), que possuíam, no mínimo, segundo grau completo.

Nessa etapa, as orientações para resposta aos itens eram lidas junto com o respondente e, em seguida, eram feitas perguntas que buscavam avaliar, com base em um roteiro de entrevista, a clareza da linguagem empregada no desenvolvimento da versão inicial do questionário. Os comentários e sugestões eram anotados em um protocolo de registro. Após tal processo, pequenos ajustes no instrumento, nas orientações para resposta, na redação de alguns itens e no formato do questionário foram realizados.

Após a validação semântica, os itens constantes do instrumento de auto-eficácia geral foram submetidos à apreciação de três professores do Instituto de Psicologia da Universidade de Brasília. Este processo foi realizado por meio de entrevista individual e envolveu tanto a análise de problemas relativos à clareza, ambigüidade das instruções e dos itens, como também a investigação da coerência dos itens em relação ao construto de auto-eficácia. Ainda nesta etapa, procedeu-se à aplicação do instrumento em 22 alunos de Pós-Graduação em Psicologia da Universidade de Brasília. A tarefa dos participantes consistia em distinguir, entre os 15 itens que compunham o questionário de auto-eficácia geral e outros 12 integrantes de uma escala de locus de controle, todos distribuídos aleatoriamente, quais deles eram indicadores de cada um dos construtos. Foram considerados confiáveis os itens corretamente classificados, por $70 \%$ ou mais de juízes, como pertencentes ao conceito em questão.

Mais algumas pequenas modificações foram feitas a partir das sugestões desses especialistas. Validado semanticamente e por juízes, o instrumento de auto-eficácia geral, então composto 15 itens associados à escala mencionada anteriormente, foi submetido a um processo de validação empírica. Para tanto, o instrumento foi aplicado em participantes de treinamentos oferecidos por três organizações, sediadas no DF, do setor terciário: prestadoras de serviços de telecomunicações $(n=581)$, administração aeroportuária $(n=99)$ e financeira $(n=1060)$. Ao final da coleta de dados e após a retirada de casos e valores extremos, obteve-se uma amostra de 1.845 treinandos com as seguintes características predominantes: sexo feminino $(63,5 \%)$, idade entre 21 e 30 anos (45\%), $3^{\circ}$ grau incompleto $(44 \%)$, solteira $(46,1 \%)$, sem filhos $(50,8 \%)$, católica $(57 \%)$, praticante de religião $(54 \%)$ e vinculada à instituição financeira (56,3\%).

Essa aplicação do instrumento, nas três organizações mencionadas, ocorria no primeiro dia dos cursos avaliados, conjuntamente com questionários que colhiam informações acerca das fontes controladoras de determinadas situações enfrentadas pelos treinandos (locus de controle), suas expectativas de suporte à transferência pós-treinamento e alguns dados sócio-demográficos. A aplicação era coletiva e os questionários eram respon- 
Meneses, P. P. M. \& Abbad, G. S. (2010). Construção e Validação de um Instrumento para Avaliar Auto-Eficácia em Situações de Treinamento, Desenvolvimento e Educação de Pessoas.

didos por todos os participantes antes de o instrutor dar início à exposição dos conteúdos e após breve explicação sobre a pesquisa pelos aplicadores.

As respostas válidas aos 15 itens de auto-eficácia foram, então, primeiramente submetidas a análises descritivas e de cunho exploratório, a fim de avaliar, no arquivo de dados, a distribuição de dados omissos, características das distribuições de frequiência das variáveis, identificação de casos extremos. Além disso, os pressupostos de normalidade, multicolineariedade e singularidade, requeridos pela análise fatorial, foram devidamente investigados.

Em seguida, foram realizadas análises dos componentes principais (Principal Components), para estimar o número de fatores e a fatorabilidade da matriz de correlações. Essa última foi avaliada pelo índice Keiser-MeyerOlkin (KMO) e pela inspeção das medidas de adequação da amostra (MAS). Utilizaram-se os seguintes critérios para a tomada de decisão referente à quantidade de fatores a serem extraídos: eigenvalues superiores a 1; análise do scree plot; porcentagem mínima de $3 \%$ de variância explicada pelo componente; cargas fatoriais superiores a 0,30 .

Por fim, efetuaram-se análises fatoriais exploratórias (Principal Axis Factoring), a fim de definir a estrutura fatorial do instrumento. Foram considerados, para constituição da versão empiricamente validada do instrumento, somente itens com cargas fatoriais superiores a 0,30 , teoricamente interpretáveis e com índices de confiabilidade superiores a 0,70 . Utilizou-se a técnica de rotação ortogonal. Em ambas as análises, como a quantidade de respostas em branco aos 15 itens analisados não ultrapassou a margem de 5\%, adotou-se o tratamento pairwise para dados omissos, conforme recomendam Tabachnick e Fidell (2001). Os resultados deste processo de validação empírica são descritos na seção seguinte.

\section{Resultados}

Entre as respostas dos 1845 participantes da amostra aos 15 itens do instrumento de auto-eficácia geral, foram localizados 149 valores extremos univariados e 29 casos considerados valores extremos multivariados. A retirada desses casos do arquivo de dados não modificou os resultados da $P C$ e $P A F$. Por esse motivo, as análises finais foram realizadas com o arquivo completo constituído pelos 1845 casos referidos. Visto que as respostas em branco não ultrapassaram 5\% do tamanho da amostra, selecionou-se o tratamento pairwise para dados omissos.

Em relação à normalidade da distribuição das respostas, os itens cujas redações consistiam afirmativas $(1,2$, $3,4,5,6,12,13$ e 14) apresentaram os maiores índices de assimetria e achatamento. Assim, procedeu-se à reflexão, no caso de índices negativos de assimetria, e a transformações de tais distribuições, utilizando-se os métodos raiz quadrada e função logarítmica de base 10 . Apesar da utilização dos métodos citados ter reduzido o valor de assimetria e achatamento para as variáveis, as soluções fatoriais não apresentaram melhorias significativas. Assim, optou-se pelo uso das variáveis originais, não transformadas para a realização das análises $P C \mathrm{e}$ PAF.

A análise dos componentes principais indicou que a matriz de dados era fatorável $(K M O=0,84)$ e sugeriu uma estrutura uni ou bifatorial. Utilizando os critérios preestabelecidos para tomada de decisão sobre a quantidade de fatores a serem extraídos, entre eles, o teste do scree plot, optou-se, em princípio, pelo teste da estrutura composta por dois fatores. A Figura 1 apresenta os eigenvalues associados a cada componente da escala de auto-eficácia.

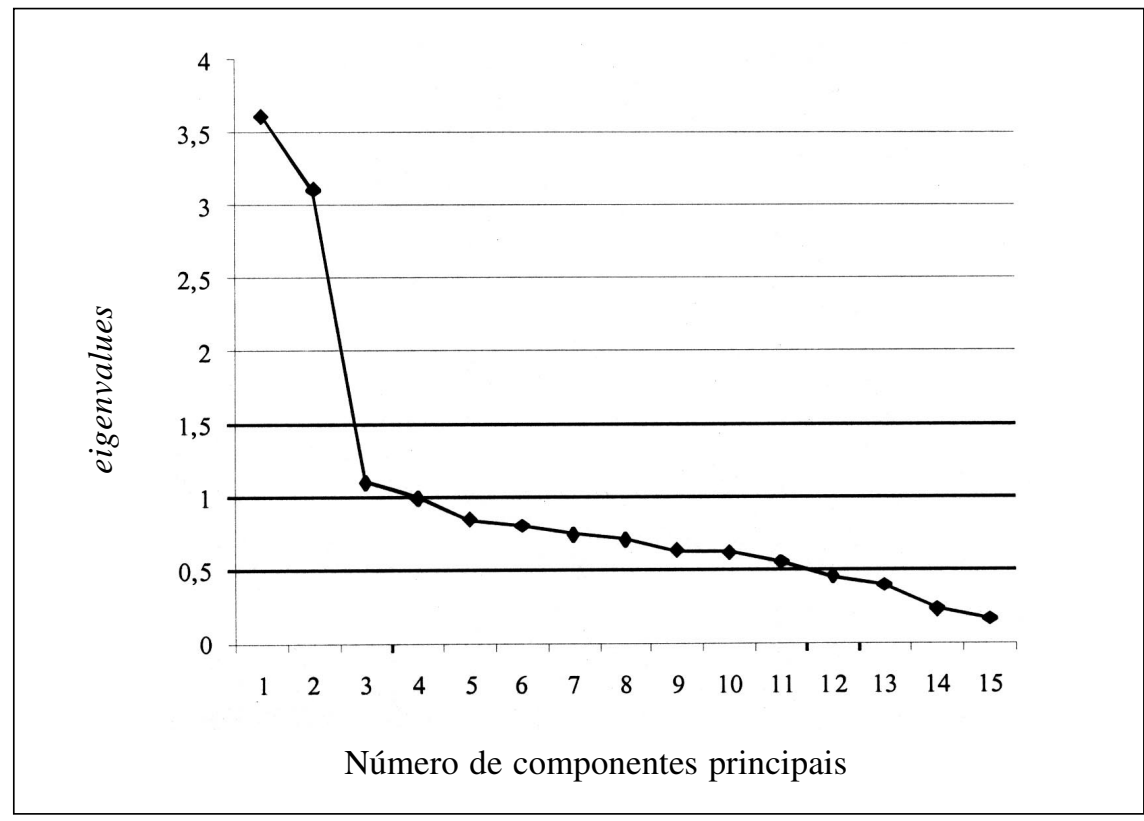

Figura 1. Teste do scree plot 
Na Tabela 1, encontra-se a estrutura empírica do instrumento de auto-eficácia com dois fatores que, conjuntamente, explicaram $38,29 \%$ da variância total das respostas. Como observado, o primeiro fator de autoeficácia, composto pelos quatro itens de conteúdo desfavorável, explicou $19,69 \%$ da variância total das respostas. A confiabilidade (alpha de Cronbach) obtida para este fator foi de 0,91 . O segundo fator de auto-eficácia, constituído por nove itens, explicou $18,60 \%$ da variância total das respostas obtidas e atingiu um índice de confiabilidade de 0,78. Dois itens (11 e 15) apresentaram cargas fatoriais inferiores a 0,30 e pequenas comunalidades e, portanto, foram excluídos das escalas. Quando a rotação oblíqua foi solicitada, pôde ser observada uma fraca e não significativa correlação $(r=-0,09)$ entre os fatores extraídos e, desta maneira, a rotação ortogonal foi a técnica empregada.

Tabela 1

Estrutura Empírica Bifatorial de Auto-Eficácia

\begin{tabular}{|c|c|c|c|}
\hline \multirow[b]{2}{*}{ Itens } & Fator 1 & Fator 2 & $h^{2}$ \\
\hline & $\begin{array}{l}\text { Cargas } \\
\text { Fatoriais }\end{array}$ & \multicolumn{2}{|l|}{$\begin{array}{l}\text { Cargas } \\
\text { Fatoriais }\end{array}$} \\
\hline $\begin{array}{l}\text { 5. Sinto-me capaz de lidar bem com a maioria dos problemas } \\
\text { que aparecem na minha vida. }\end{array}$ & & 0,65 & 0,42 \\
\hline 4. Lido bem com problemas inesperados. & & 0,63 & 0,39 \\
\hline 2. Confio nas minhas habilidades. & & 0,61 & 0,38 \\
\hline 14. Recupero-me rapidamente depois de um fracasso. & & 0,59 & 0,35 \\
\hline 1. Sou capaz de realizar com sucesso meus planos de vida. & & 0,54 & 0,29 \\
\hline 6. Encaro dificuldades como um desafio. & & 0,56 & 0,31 \\
\hline 3. Quando decido fazer algo, logo parto para a ação. & & 0,45 & 0,21 \\
\hline 13. Posso dizer que na vida tive mais sucessos que fracassos. & & 0,43 & 0,19 \\
\hline 12. Mesmo que comece mal uma atividade, posso finalizá-la com sucesso. & & 0,40 & 0,16 \\
\hline 7. Eu desisto facilmente daquilo que me proponho a fazer. & 0,89 & & 0,81 \\
\hline 8. Se algo parece muito complicado, eu nem tento realizá-lo. & 0,89 & & 0,80 \\
\hline 9. Sinto-me inseguro diante de situações novas. & 0,71 & & 0,50 \\
\hline 10. Deixo-me abater diante de fracassos. & 0,87 & & 0,76 \\
\hline
\end{tabular}

11. Sinto-me incapaz de realizar uma nova atividade sem a ajuda de instruções.

15. Sinto-me incapaz de iniciar uma atividade sem o encorajamento de outras pessoas.

\begin{tabular}{lcccc}
\hline Fatores & Eigenvalues & \% Variância & $\begin{array}{c}\alpha \text { de Cronbach } \\
\text { Explicada }\end{array}$ & $\begin{array}{c}\text { Número } \\
\text { de Itens }\end{array}$ \\
\hline F1. Auto-eficácia p/ itens desfavoráveis & 3,47 & 19,69 & 0,91 & 4 \\
F2. Auto-eficácia p/itens favoráveis & 3,24 & 18,60 & 0,78 & 9 \\
\hline
\end{tabular}

Obtida a estrutura fatorial do instrumento de auto-eficácia geral, na seção seguinte discute-se a qualidade psicométrica das escalas, o perfil demográfico dos participantes em relação aos fatores de auto-eficácia, bem como algumas implicações teóricas, metodológicas e práticas desta pesquisa.

\section{Discussão dos Resultados}

Em relação à formatação, na tentativa de tornar o processo de avaliação mais atraente para o respondente, optou-se pela construção de um instrumento de pesquisa reduzido na quantidade de itens e genérico em sua abrangência, o que, respectivamente, demandaria menos esforço cognitivo dos treinandos e auxiliaria na classificação de resultados de treinamentos. Tal classificação permitira o agrupamento de eventos instrucionais por intensidade e abrangência de seus efeitos sobre autoeficácia, além de dificultar a rotulação dos avaliados a partir de seus níveis no construto em questão.

Geralmente, os instrumentos integrantes de sistemas de avaliação de treinamento possuem uma quantidade de itens que torna entendiante a tarefa do avaliador e, conseqüentemente, eleva-se a probabilidade de ocorrência de erros como leniência ou halo, por exemplo. Esta foi a principal justificativa para a construção de um instrumento de pesquisa com apenas 15 itens em sua versão inicial. Apesar de a qualidade psicométrica de um determinado instrumento depender, pelo menos em parte, da quantidade de itens que o constituem, os resultados do processo de validação teórica, semântica e por juízes, e empírica revelou a adequação das escalas desenvolvidas. 
No que diz respeito ao processo de validação teórica, as entrevistas realizadas com os 15 profissionais das organizações pesquisadas e os três professores da Instituição de Ensino Superior mencionada, bem como a aplicação coletiva das escalas em 22 alunos de pós-graduação, demandaram pequenos ajustes no instrumento, efetivados anteriormente à coleta de dados para a validação empírica. Essa, por sua vez, revelou a validade e a confiabilidade das escalas constituídas, haja vista os índices estatísticos obtidos. Neste caso, vale lembrar que a menor carga fatorial encontrada foi de 0,40 e a maior, de 0,89 nas escalas finais. Cada uma das escalas obtidas explicou, separadamente, aproximadamente $19 \%$ da variância total obtida. Também foram satisfatórios os índices de confiabilidade, igual a 0,78 para a escala de auto-eficácia para itens favoráveis, constituída por nove itens, e igual a 0,91 para a escala constituída pelos quatro itens de conteúdos desfavoráveis.

Neste ponto, um aspecto merece atenção especial. Como observado, a escala com a menor quantidade de itens alcançou um maior índice de precisão e confiabilidade, mesmo com os itens tendo sido construídos de forma desfavorável, o que, em princípio, dificultaria a tarefa do respondente. Ao contrário, a escala constituída pelos nove itens elaborados de forma favorável alcançou um índice de confiabilidade apenas mediano. Possíveis explicações para o ocorrido remetem ao contexto da pesquisa e às características da amostra estudada.

Considerando que a base de uma análise fatorial consiste na matriz de correlações entre os itens de determinado instrumento (intercorrelações), o fato de o fator com menor quantidade de itens ter resultado em índices de validade maiores pode contribuir para a discussão da estrutura bifatorial obtida, a qual, teoricamente, não faz sentido. Caso analisadas algumas estatísticas descritivas, constata-se que o desvio-padrão do fator com menor quantidade de itens é menor $(D P=0,44)$ se comparado ao fator com mais itens $(D P=1,37)$, como era de se esperar.

Os participantes, dito de outra maneira, responderam de maneira mais coesa aos itens que representavam o conceito de auto-eficácia de forma desfavorável do que aqueles a eles apresentados favoravelmente. Isso talvez indique que, nas organizações onde a pesquisa de validação fora realizada, os indivíduos talvez não possam demonstrar explicitamente sensações de incapacidade. Mas se trata apenas de uma hipótese ante os resultados estatísticos obtidos. Pesquisas que relacionem os indicadores de auto-eficácia desenvolvidos com os tipos motivacionais de valor originalmente propostos por Schwartz e Blisky (1990), sobretudo o de realização, fazem-se importantes à medida que podem testar tal hipótese.

Outra hipótese refere-se ao fato de que a escala de autoeficácia era aplicada no primeiro dia de treinamento, juntamente com outros instrumentos, entre eles, um de locus de controle, recentemente validado com a mesma amos- tra utilizada neste estudo (Meneses, 2002), que avaliava atribuições individuais de responsabilidade sobre eventos cotidianos. Segundo os resultados obtidos mediante análises estatísticas, como as apresentadas nesta pesquisa, duas escalas foram constituídas, a primeira, referente à fonte externa de controle e a segunda, à fonte interna. $\mathrm{O}$ interessante é que as cargas fatoriais mais elevadas foram observadas no fator externalidade, indicando que os indivíduos, também em sua maior parte pertencente à instituição financeira, do sexo feminino, com idade até 30 anos, $3^{\circ}$ grau incompleto, sem filhos e católicos creditavam o sucesso de suas ações a fontes como superiores, chefes e colegas de trabalho ou mesmo à sorte.

Talvez, pelas características também da própria organização, de natureza financeira, estes indivíduos tenham se acostumado com o fato de pouco poderem fazer para modificar os resultados de alguma ação empreendida. Caso aceita, esta hipótese justificaria as cargas fatoriais elevadas no fator auto-eficácia para itens desfavoráveis, no sentido de que as pessoas possam ter aprendido a reagir mais eficazmente diante de situações além da alçada delas próprias, onde o controle encontra-se, talvez, nas mãos de indivíduos hierarquicamente superiores.

Formulada esta hipótese, decidiu-se pela realização de um teste $t$ para amostras independentes, na tentativa de se investigar o nível de auto-eficácia dos participantes nas duas escalas obtidas no presente estudo poderia ser decorrente, entre outros aspectos, da filiação institucional. A amostra de respondentes foi então dividida em dois grupos, de forma que aproximadamente cada um deles fosse constituído por aproximadamente metade dos casos. Do primeiro grupo participaram os respondentes vinculados à instituição financeira $(60 \%$ dos casos válidos), ao passo que os indivíduos pertencentes às empresas dos setores de telecomunicação e de administração aeroportuária formaram o segundo grupo (40\% dos casos válidos).

Os resultados da análise mencionada indicaram diferenças significativas entre as médias dos respondentes dos grupos constituídos para os dois fatores de auto-eficácia. Em relação ao primeiro fator (auto-eficácia para itens desfavoráveis), a média obtida a partir das respostas, em uma escala de 1 a 5, dos participantes do grupo 1 (instituição financeira) foi de 1,85 , enquanto a média encontrada para os grupo 2 (telecomunicações e administração aeroportuária) foi de 3,70. Quanto ao segundo fator (auto-eficácia para itens favoráveis), a média do grupo 1 foi de 4,26, ao passo que a do grupo 2, de 4,51. Para os dois fatores, considerando intervalos de confiança de $95 \%$, as diferenças entre as médias descritas foram significativas, como apresentado na Tabela 2.

Como descrito, os participantes afiliados à instituição financeira apresentaram níveis mais elevados de autoeficácia para situações desfavoráveis, bem como níveis menores para situações favoráveis, indicando que, como na pesquisa acerca das fontes de controle de suas ações, 
Tabela 2

Resultados dos Testes $t$

\begin{tabular}{|c|c|c|c|c|c|c|c|}
\hline \multirow{2}{*}{ Variáveis } & \multirow{2}{*}{ Fatores } & \multicolumn{3}{|c|}{ Estatísticas Descritivas } & \multirow{2}{*}{$t$} & \multirow{2}{*}{ Significância } & \multirow{2}{*}{$\begin{array}{c}\text { Diferença } \\
\text { entre médias }\end{array}$} \\
\hline & & $N$ & Média & Desvio-Padrão & & & \\
\hline \multicolumn{8}{|l|}{ Empresa } \\
\hline 1. financeira & Itens & 1000 & 1,85 & 0,70 & \multirow{2}{*}{$-31,77$} & \multirow{2}{*}{0,000} & \multirow{2}{*}{$-1,84$} \\
\hline 2. outras & Desfavoráveis & 700 & 3,70 & 1,40 & & & \\
\hline \multicolumn{8}{|l|}{ Empresa } \\
\hline 1. financeira & Itens & 1000 & 4,26 & 4,26 & \multirow{2}{*}{12,80} & \multirow{2}{*}{0,000} & \multirow{2}{*}{$-0,25$} \\
\hline 2. outras & Desfavoráveis & 700 & 4,51 & 4,51 & & & \\
\hline
\end{tabular}

devido às configurações de trabalho da organização de origem, tais participantes aprenderam a lidar mais enfaticamente com situações desfavoráveis de trabalho. De acordo com as médias e os desvios-padrões obtidos, podese afirmar que os profissionais da instituição financeira apresentaram níveis mais elevados de auto-eficácia quando os itens justamente indicavam, antes de serem respondidos, baixos níveis de auto-eficácia, do que aqueles provenientes de outras organizações.

Apesar de confirmada a hipótese, de que a filiação institucional seria responsável pela obtenção de uma estrutura empírica com dois fatores de auto-eficácia, talvez outras características das organizações estudadas, ou mesmo outras características dos próprios respondentes, tenham impactado na forma como os indivíduos reagiram diferenciadamente ante os itens de conteúdo desfavorável. De qualquer forma, independentemente das explicações desenvolvidas para a extração de uma estrutura bifatorial, merece atenção o fato de que o instrumento mostrou-se válido e confiável.

\section{Conclusão}

O instrumento de Auto-Eficácia Geral mostrou-se bastante claro e consistente; recebeu relativamente poucas críticas e sugestões de mudanças durante os processos de análise semântica, por juízes e empírica, os quais, por sua vez, confirmaram a validade, a precisão e a confiabilidade do instrumento. A fim de confirmar a estrutura obtida no presente estudo, recomenda-se que novas análises fatoriais exploratórias sejam feitas após os itens, cujas redações indicavam baixos níveis de autoeficácia, serem novamente elaborados, conforme o mesmo padrão utilizado na confecção dos demais itens do instrumento. Ou ainda, também com o intuito de testar a estabilidade da estrutura bifatorial ora obtida, sugere-se a aplicação do instrumento, na versão descrita neste estudo, em amostras de indivíduos e organizações diversificadas.

Apesar dos resultados terem indicado a qualidade do instrumento em voga, a variância total explicada pelos dois fatores estabelecidos, de aproximadamente $40 \%$, indica a existência de outros aspectos relacionados ao construto não abarcados pelo instrumento. Talvez a desconsideração de aspectos relacionados ao autoconceito tenha sido responsável pela taxa de variância explicada pelos dois fatores emergidos. Desta maneira, estudos posteriores, quando oportuno, devem preocupar-se em investigar as relações entre os itens do instrumento apresentado e outros relativos ao autoconceito, a fim de distinguirem, empiricamente, a associação dos itens aos construtos referentes.

Na presente pesquisa, o processo de validação por juízes permitiu com que os itens de auto-eficácia fossem distinguidos de outros relativos ao locus de controle. Entretanto, distinção alguma foi proposta entre os 15 itens de auto-eficácia e outros construtos. Estudos que investiguem a associação de auto-eficácia com outros construtos correlatos, tais como auto-estima, autoconfiança e autoconceito, nesse sentido, permitirão uma compreensão mais ampla do conceito em questão. Mais uma vez, torna-se necessária a aplicação do instrumento de auto-eficácia em amostras diferenciadas, a fim de garantir uma maior generalidade dos dados.

A fim de se compreender a relação entre auto-eficácia e resultados de treinamento, seria interessante, também, que pesquisas posteriores busquem investigar a capacidade de a auto-eficácia explicar satisfação, aprendizagem e desempenho individual pós-treinamento. Nos Estados Unidos, local onde surgiram as primeiras tentativas de se investigar essa relação, a autoeficácia, como discutido, consiste importante característica individual explicativa de resultados de TD\&E. No Brasil, estudos desta natureza ainda precisam ser realizados mais sistematicamente, tanto porque, diferentemente dos Estados Unidos, a cultura brasileira parece destacar a coletividade e não a individualidade. Será, assim, que construtos auto-referentes, fundamentados na noção do self, são de fato tão relevantes como características do contexto de trabalho - o apoio de superiores, pares e subordinados - na explicação de resultados de ações de TD\&E? 
Meneses, P. P. M. \& Abbad, G. S. (2010). Construção e Validação de um Instrumento para Avaliar Auto-Eficácia em Situações de Treinamento, Desenvolvimento e Educação de Pessoas.

\section{Referências}

Abbad, G. (1999). Um modelo integrado de avaliação do impacto do treinamento no trabalho - IMPACT. Tese de Doutorado não-publicada, Universidade de Brasília, DF.

Abbad, G., Pilati, R., \& Pantoja, M. J. (2003). Avaliação de treinamento: Análise da literatura e agenda de pesquisa. Revista de Administração da USP, 38, 205-218.

Azzi, R. G., \& Polydoro, S. A. J. (Eds.). (2006). Auto-eficácia em diferentes contextos. Campinas, SP: Alínea

Bandura, A. (1988). Self-regulation of motivation and action through goal systems. In V. Hamilton, G. H. Bower, \& N. H. Frijda (Eds.), Cognitive perspectives on emotion and motivation (pp. 37-61). Dordrecht, Netherlands: Kluwer Academic.

Bandura, A. (1989). Perceived self-efficacy in the exercise of personal agency. The Psychologist: Bulletin of the British Psychological Society, 2, 411-424.

Borges-Andrade, K. E. (1997). Treinamento de pessoal: Em busca do conhecimento e tecnologia relevantes para as organizações brasileiras. In A. Tamayo, J. E. BorgesAndrade, \& W. Codo (Eds.), Trabalho, organizações e cultura (pp. 129-149). São Paulo, SP: Cooperativa de Autores Associados.

Bouffard-Bouchard, T. (1990). Influence of self-efficacy on performance in a cognitive task. Journal of Social Psychology, 130(3), 353-363.

Britto, M. J. P. (1999). Avaliação de impacto de treinamentos na área de reabilitação: Preditores individuais e situacionais. Dissertação de Mestrado não-publicada, Universidade de Brasília, DF.

Brockner, J. (1988). Self-esteem at work: Research, theory, and practice. Lexington, MA: Lexington Books.

Brown, T. C., \& Latham G. P. (2000). The effects of goal setting and self-instruction training on the performance of unionized employees. Industrial Relation, 55, 80-94.

Cervone, D., \& Scott, W. D. (1995). Self-efficacy theory of behavioral change: Foundations, conceptual issues, and therapeutic implications. In W. O'Donohue \& L. Krasner (Eds.), Theories of Behavior therapy: Exploring behavior change (pp. 349-383). Washington, DC: American Psychological Association.

Coelho, F. A., Jr. (2004). Avaliação de treinamento a distância: Suporte à aprendizagem e impacto do treinamento no trabalho. Dissertação de Mestrado não-publicada, Universidade de Brasília, DF.

Colquit, J. A., LePine, J. A., \& Noe, R. A. (2000). Toward an integrative theory of training motivation: A meta-analytic path analysis of 20 years of research. Journal of Applied Psychology, 85(5), 678-707.

Eden, D., \& Kinnar, J. (1991). Modeling Galatea: Boosting self-efficacy to increase volunteering. Journal of Applied Psychology, 76(6), 770-780.

Ford, J. K., Quiñones, M. A., Sego, D. J., \& Sorra, S. J. (1992). Factors affecting the opportunity to perform trained tasks on the job. Personnel Psychology, 45(3), 511-527.

Freitas, I. A., \& Borges-Andrade, J. E. (2004). Efeitos de treinamentos nos desempenhos individual e organizacional. Revista de Administração de Empresas, 44(3), 44-56.

Gist, M. E. (1989). The influence of training method on selfefficacy and idea generation among managers. Personnel Psychology, 42, 787-805.
Gist, M. E., \& Mitchell, T. R. (1992). Self-efficacy: A theoretical analysis of its determinants and malleability. The Academy of Management Review, 17(2), 183-211.

Goldstein, I. L. (1991). Training in work organizations. In M. Dunnette \& L. Hough (Eds.), Handbook of industrial and organizational psychology (pp. 507-619). Palo Alto, CA: Consulting Psych.

Kirkpatrick, D. L. (1976). Evaluation of training. In R. L. Craig (Ed.), Training and development handbook. New York: McGraw-Hill.

Latham, G. P., \& Frayne, C. A. (1989). Self-management training for increasing job attendance: A follow-up and a replication. Journal of Applied Psychology, 74(3), 411-416.

Lummertz, J. G., \& Biaggio, A. M. B (1986). Relações entre autoconceito e nível de satisfação familiar em adolescentes. Arquivos Brasileiros de Psicologia, 38(2), 158-166.

Marx, R. D. (1982). Relapse prevention of managerial training: A model for maintenance of behavior change. Academy of Management Review, 7, 433-441.

Mathieu, J. E., Martineau, J. W., \& Tannenbaum, S. I. (1993). Individual and situational influences on the development of self-efficacy: Implications for training effectiveness. Personnel Psychology, 46(1), 125-147.

Maurer, T. J., \& Pierce, H. R. (1988). A comparison of Likert Scale and traditional measures of self-efficacy. Journal of Applied Psychology, 83(2), 324-329.

Meneses, P. P. M. (2002). Auto-eficácia, locus de controle, suporte à transferência e impacto do treinamento no trabalho. Dissertação de Mestrado não-publicada, Universidade de Brasília, DF.

Morin, L., \& Latham, G. P. (2000). The effect of mental practice and goal setting as a transfer of training intervention on supervisor's self-efficacy and communication skills: An exploratory study. Applied Psychology, 49, 566-578.

Noe, R. A. (1986). Trainee's attributes and attitudes: Neglected influences on training effectiveness. Academy of Management Review, 11(4), 736-749.

Page, B. J., \& Hulse-Killacky, D. (1999). Development and validation of the corrective feedback self-efficacy instrument. The Journal for Specialists in Group Work, 24(1), 37-54.

Philips, P. P., \& Philips, J. J. (2001). Symposium on the evaluation of training. International Journal of Training and Development, 5(4), 240-247.

Salas, E., \& Cannon-Bowers, A. (2001). The science of training: A decade of progress. Annual Review of Psychology, 52, 471-499.

Sallorenzo, L. H. (2000). Avaliação de impacto de treinamento no trabalho: Analisando e comparando modelos de predição. Dissertação de Mestrado não-publicada, Universidade de Brasília, DF.

Schwartz, H., \& Blisky, W. (1990). Toward a theory of the universal content and structure of values: Extensions and cross-cultural replications. Journal of Personality and Social Psychology, 58, 878-891.

Sherer, M., \& Adams, C. H. (1983). Construct validation of the Self-Efficacy Scale. Psychological Reports, 53, 899-902.

Tabachnick, B. G., \& Fidell, L. S. (2001). Using multivariate statistics. New York: Harper \& Row.

Tamayo, N. (2002). Autoconceito profissional, suporte à transferência e impacto do treinamento no trabalho. Dissertação de Mestrado não-publicada, Universidade de Brasília, DF.

Tannenbaum, S. I., \& Yukl, G. (1992). Training and development in work organizations. Annual Review of Psychology, 43, 399-441. 
Tannenbaum, S. I., Mathieu, J. E., Salas, E., \& Canon-Bowers, J. A. (1991). Meeting trainee's expectations: The influence of training fulfillment on the development of commitment, self-efficacy, and motivation. Journal of Applied Psychology, 76(6), 759-769.

Taylor, M. S., Locke, E. A., Lee, C., \& Gist, M. E. (1984). Type a behavior and faculty research productivity: What are the mechanisms? Organizational Behavior \& Human Performance, 34,402-418.

Vroom, V. H. (1995). Work and motivation. San Francisco: Jossey-Bass.

Zerbini, T. (2003). Estratégias de aprendizagem, reações de um curso via internet, reações ao tutor e impacto do treinamento no trabalho. Dissertação de Mestrado não-publicada, Universidade de Brasília, DF.

Warr, P., Allan, C., \& Birdi, K. (1999). Predicting three levels of training outcome. Journal of Occupational and Organizational Psychology, 72(3), 351-375.

Wood, R. E., \& Locke, E. A. (1987). The relation of self-efficacy and grade goals to academic performance. Educational and Psychological Measurement, 47(4), 1013-1024. 